\title{
Generation of time synchronized frequency tunable picosecond light pulses in a mode-locked $\mathrm{Nd}$ :glass double laser system
}

\author{
J. Furthner, H. Schillinger' and A. Penzkofer \\ Naturwissenschaftliche Fakultät II-Physik, Universität Regensburg, D-8400 Regen.sburg. Fìd. Re'p. Germany'
}

Received 30 March 1990

\begin{abstract}
An active and passive mode-locked $\mathrm{Nd}$ :glass double laser system is built-up. It consists of two gain branches and a common antiresonant ring. The electro-optic Q-switch, the acousto-optic modulator and the saturable absorber are incorporated in the antiresonant ring. A Nd:phosphate glass rod, a Nd: silicate glass rod, and birefringent tuning elements are in the separate gain branches. Time synchronized pulses of 15 ps duration were be tuned over a frequency region of 0 to $200 \mathrm{~cm}^{-1}$.
\end{abstract}

\section{Introduction}

Time synchronized frequency tunable picosecond light pulses are needed in many time resolved spectroscopic experiments (e.g. CARS studies, all kinds of pump and probe techniques) $[1-5]$. Fixed-frequency, time-synchronized picosecond light pulses have been obtained by harmonics generation [6-8], stimulated Raman scattering [6,7,9-11], and Stokesanti-Stokes Raman interaction [11-13]. Frequency tunable time-synchronized ultrashort light pulses have been generated in parametric generator-amplifier systems [14-16], laser generator-amplifier systems (amplified spontaneous emission) [17-23], and synchronous pumped lasers [24-28]. The synchronous pumping of two dye lasers with a modelocked pump laser allowed the independent frequency tuning of both dye lasers $[25,26]$. In some pump and probe experiments intense laser pulses are combined with picosecond or femtosecond light continua (for reviews see $[29,30]$ ).

In this paper we describe a mode-locked $\mathrm{Nd}$ :glass double laser system which allows the time-synchronized generation of two frequency tunable pulse trains. The tuning range is limited by the spectral luminescence width of the two applied Nd:glass rods.

\footnotetext{
1 Present address: Max Planck Institut für Quantenoptik, D.
} 8406 Garching, Fed. Rep. Germany
Intense picosecond light pulses of 15 ps duration and a frequency difference between 0 and $200 \mathrm{~cm}^{-1}$ have been generated.

\section{Experimental}

The experimental setup of the time-synchronized, frequency tunable double-pulse laser is shown in fig. 1. The system consists of two laser branches with a common antiresonant ring. The resonator branches have separate gain media (Nd: phosphate glass Schott LG703 and Nd: silicate glass Schott LG 680) and frequency tuning elements (birefringent filters [31]). The common antiresonant ring (Sagnac interferometer (32-34]) incorporates an electro-optic Qswitch consisting of a half-wave voltage double Pockels cell and two stacked-Brewster plate polarizers [35], an acousto-optic modulator for active loss modulation [36], and a saturable absorber for passive mode-locking [37]. The common Q-switching, active, and passive mode-locking in the antiresonant ring leads to the time-synchronized simultaneous generation of two picosecond pulse trains which are frequency tuned independently by the birefringent filters in the gain branches. The Nd:phosphate glass laser is pumped by two Xe flashlamps in series which are connected to a capacitor bank of $500 \mu \mathrm{F}$. The $\mathrm{Nd}$ : silicate glass laser is operated with two Xe flash- 


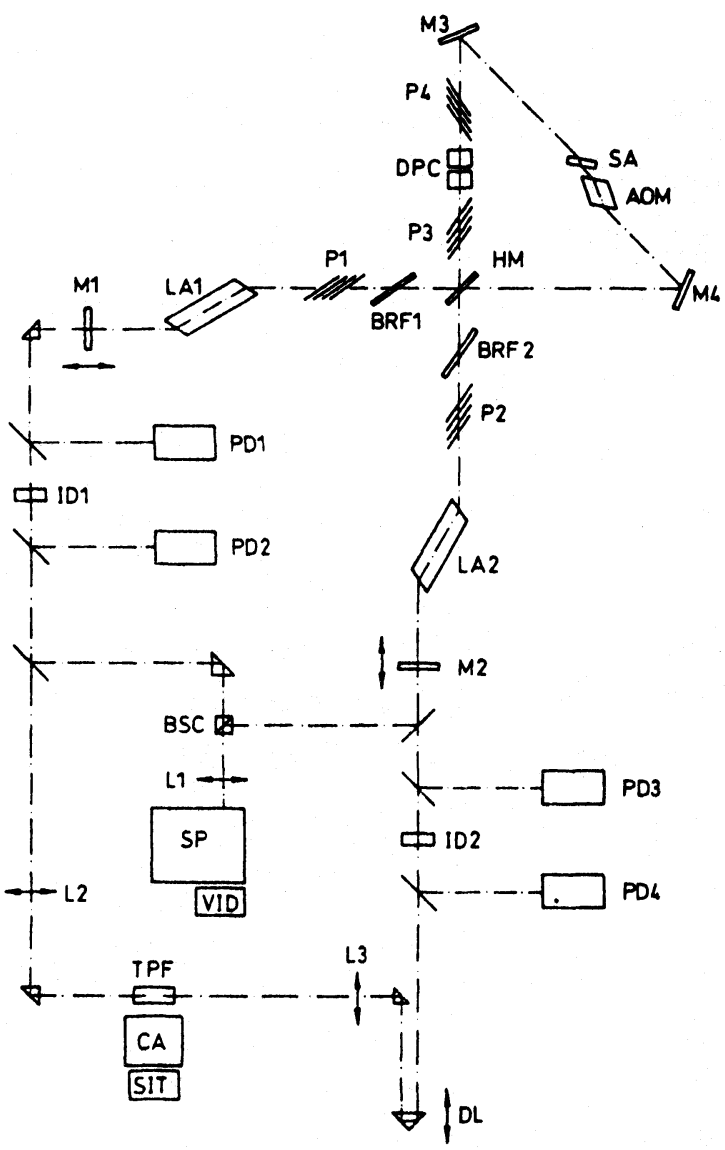

Fig. 1. Experimental setup of branched picosecond double laser system. M1. M2: output mirrors (reflectivity $70 \%$, curvature 3 m). M3, M4: plane mirrors (100\% reflectivity). LA1: Nd:phosphate glass laser rod (Schott type LG703). LA2: Nd: silicate glass laser rod (Schott type LG680). P1-P4, stacked glass plate polarizers. BRF1, BRF2: single plate birefringent filters (quartz plates, thickness $5 \mathrm{~mm}$ ). DPC: $K D^{*} P$ half-wave voltage double Pockels cell (Gsänger type LM81M, Q-switch driver: Baasel type EOQS2). SA: saturable absorber in center of anti-resonant ring (Kodak dye No. A9740; small-signal singlepath transmission $T_{0}=0.7$ ). AOM: acoustic-optic modulator (IntraAction model ML-50Q mode-locker and model MLE-6A mode-locker driver; $50 \mathrm{MHz}$ acoustic frequency, $325 \mathrm{kHz}$ modespacing). HM: $50 \%$ beamsplitter (actual reflectivity 47 to $49 \%$ within tuning range, false coupling for Nd:phosphate laser ca. $5 \%$. false coupling for Nd: phosphate laser ca. 3\%). PDI-PD4: photodetectors. ID1, ID2: saturable absorbers (Kodak dye No. A9860. small signal transmission $17.3 \%$ ) for peak intensity detection [54]. L1-L3: lenses. SP: grating spectrometer. VID, vidicon (OSA system of B u M Spectronik). TPF: two-photon fluorescence cell for cross-correlation detection (dye: $5 \times 10^{-3} \mathrm{molar}$ rhodamine $6 \mathrm{G}$ in methanol). CA: camera. SIT: silicon-intensified-target vidicon ( $B$ u M Spectronik). DL: optical delay line. lamps in parallel whereby each lamp is connected to a capacitor bank of $500 \mu \mathrm{F}$.

Two-branch lasers with a common antiresonant ring part have been proposed in the literature $[32,34,38]$. Colliding pulse mode-locking of picosecond [39-49] and femtosecond [50-52] lasers in antiresonant ring arrangements is a frequently used technique. In ref. [53] a frequęncy tunable picosecond laser is described which has a mechanical Qswitch, saturable absorber and acoustic-optic modelocker in the antiresonant ring.

The pulse trains of the two-branch laser are analysed. The pulse energies are registered by the photodetectors PD1 and PD3 which are calibrated by a pyroelectric energy meter (Gentec type ED100A). The peak pulse intensities are determined by energy transmission measurements through the saturable absorber cells IDI and ID2 (Kodak dye No. 9860. small signal transmission $T_{0}=0.173$ ) with the photodetector PD1, PD2 and PD3, PD4 [54]. The spectra are recorded with a $25 \mathrm{~cm}$ grating spectrometer and a vidicon.

The pulse durations are measured by the two-photon fluorescence technique using a colliding pulse arrangement (not shown in fig. 1) [55]. The half-width $\Delta z$ (fwhm) of a fuorescence trace $S(z)$ (measured at the signal height $[S(\infty)+S(0)] / 2)$ is related to the auto-correlation time $\Delta \tau$ by $\Delta \tau=2 n \Delta z / c_{0}$ and the relation between pulse duration $\Delta t_{\mathrm{L}}$ and the autocorrelation time $\Delta \tau$ is $\Delta l_{L}=\Delta \tau / \gamma, n$ is the refractive index of the two-photon absorbing dye solution at the laser frequency and $c_{0}$ is the vacuum light velocity. $\gamma$ depends on the temporal pulse shape. For gaussian pulse shapes it is $\gamma^{\prime}=2^{1 / 2}[56,57]$.

The cross-correlation time measurement of the two pulse trains is illustrated in the lower part of fig. 1. The fluorescence trace $S_{\mathrm{c}}(z)$ of the two-photon excited dye solution in the cell TPF is imaged to a SIT camera. The cross-correlation time $\Delta \tau_{\mathrm{c}}(\mathrm{fwhm})$ is related to the trace width $\Delta z_{c}$ (fwhm measured at $\left.\left[S_{\mathrm{c}}(\infty)+S_{\mathrm{c}}(0)\right] / 2\right)$ by $\Delta \tau_{\mathrm{c}}=2 n \Delta z_{\mathrm{c}} / c_{\mathrm{c}}$, and for gaussian pulses the relation between $\Delta \tau_{\mathrm{c}}$ and the pulse durations $\Delta t_{2}$ and $\Delta t_{2}$ of the two interacting pulse trains is $\Delta \tau_{c}=\left[\left(\Delta t_{1}\right)^{2}+\left(\Delta t_{2}\right)^{2}\right]^{1 / 2}$. The time jitter of the two pulse trains results in a shot to shot changing of the position $z_{p}$ of the two-photon fluorescence peak. For $N$ measurements the mean position is 


$$
\bar{z}_{\mathrm{p}}=\left(\sum_{i=1}^{N} z_{\mathrm{p}, i}\right) / N
$$

The standard deviation of the peak position is

$\sigma_{z, \mathrm{p}}=\left[\sum_{i=1}^{N}\left(z_{\mathrm{p}, i}-z_{\mathrm{p}}\right)^{2} /(N-1)\right]^{1 / 2}$,

and the temporal jitter is $\sigma_{t, p}=2 n \sigma_{z, p} / c_{0}$.

\section{Results}

The performance data of the double-laser system are determined for the active mode-locked (without saturable absorber) and the hybrid mode-locked operation (simultaneous acousto-optic and passive mode-locking).

\subsection{Total output laser energy}

The total output pulse energy of each laser branch versus the input energy to the flashlamps is plotted in fig. 2. The double laser is mode-locked acoustooptically. The Q-switch opens $600 \mu$ s after firing the lamps. With increasing pump energy the output laser

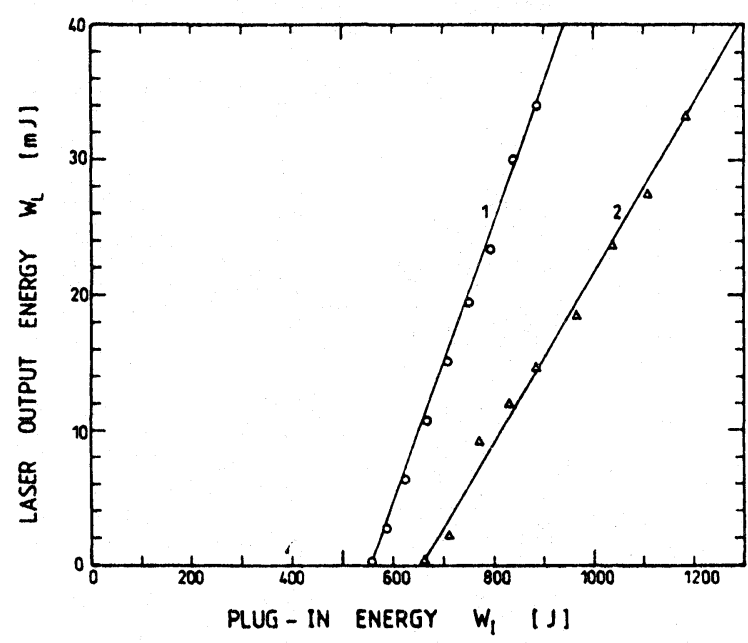

Fig. 2. Laser output energy versus plug-in input energy. Curve 1 and circles: Nd:phosphate glass laser at $\lambda_{1}=1053 \mathrm{~nm}$; curve 2 and triangles: Nd: silicate glass laser at $\lambda_{2}=1061 \mathrm{~nm}$. Laser system is active mode-locked with acousto-optic mode-locker drive power of $P_{20}=2 \mathrm{~W}$. Q-switch opening at $t_{Q}=600 \mu$ s after laser firing. energy rises linearly above the laser threshold. Many mode-locked spikes are formed with increasing pump voltage [58]. The differential plug-in slope efficiencies of both branches are [59] $\eta_{\mathrm{st}}$ (Nd:phosphate) $=1 \times 10^{-4}$ and $\eta_{\mathrm{sl}}$ ( Nd: silicate $)=6.3 \times 10^{-5}$.

The hybrid mode-locked double laser is operated slightly above the laser threshold (flashlamp pump voltage approximately $50 \mathrm{~V}$ above threshold). The saturable absorber Kodak No. $9740[60,61]$ with a single-path small signal transmission of $T_{10}=0.7$ is used for passive mode-locking. In this case only single pulse trains are formed with an output pulse train energy of approximately $5 \mathrm{~mJ}$ for each branch.

\subsection{Laser build-up time}

The laser build-up time $\iota_{\text {bu }}$ versus excess flashlamp voltage (voltage above laser threshold) is displayed by the solid curves in fig. 3 for the active mode-locked laser. $t_{\text {bu }}$ is defined as the time difference between the positions of the peak of the first laser spike and the moment of opening the electro-optic Q-switch. The jitter of the build-up time $\sigma_{t, b u}$ is shown by the dashed-dotted curves in fig. 3. At a excess flashlamp voltage of $V_{e} \approx 300 \mathrm{~V}$ the laser build-up time is shortened to approximately $800 \mathrm{~ns}$, and the build-up time jitter (dash-dotted curves) is reduced to about $20 \mathrm{~ns}$ indicating a good temporal synchronization of both laser branches.

The hybrid mode-locked double laser system (small-signal saturable absorber transmission $T_{0} \approx 0.7$ ) gives a good temporal overlap of the pulse trains of both branches already slightly above the laser threshold $\left(V_{\mathrm{e}} \approx 50 \mathrm{~V}\right)$. The laser build-up time is of the order of $3 \mu \mathrm{s}$, but the temporal jitter between the peak train positions of both branches is only a few tens of nanoseconds. The bleaching of the saturable absorber synchronizes both laser branches (high round-trip gain for both lasers within opening time of saturable absorber).

\subsection{Pulse train length}

The dependence of the pulse lengths, $t_{1 / 2}$ (fwhm, first spike) on the excess flashlamp voltage is displayed by the dashed curves in fig. 3. The double laser system is actively mode-locked. Operating the laser branches $200 \mathrm{~V}$ above the threshold voltage results 


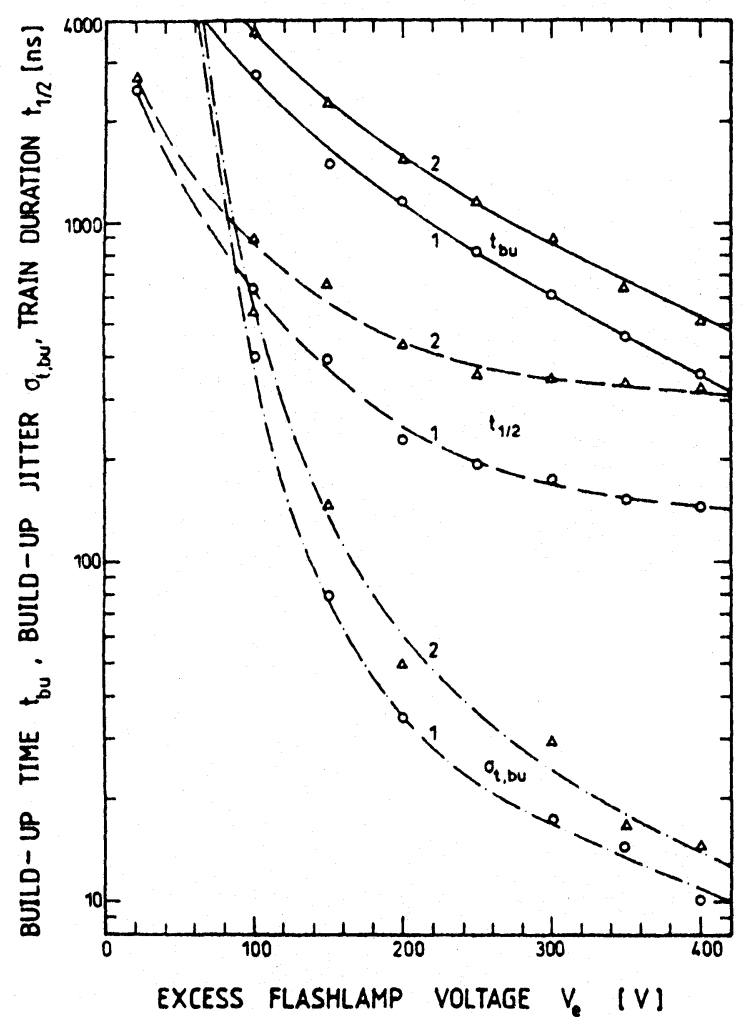

Fig. 3. Temporal behaviour of acousto-optic mode-locked double laser system. $\left(P_{\mathrm{ao}}=2 \mathrm{~W}\right)$. Curves 1 and circles belong to $\mathrm{Nd}$ :phosphate laser branch. Curves 2 and triangles belong to Nd: silicate laser branch. The flashlamp threshold voltages are $V_{\text {in }}(\mathrm{Nd}:$ phosphate $)=1490 \mathrm{~V}$ and $V_{\text {in }}$ (Nd: silicate) $=1150 \mathrm{~V}$. Solid curves: laser build-up time. Dash-dotted curves. jitter of laser build-up time. Dashed curves. pulse train width (fwhm) of first laser spike.

in a good temporal overlap of the pulse trains (pulse halfwidth $\gg$ temporal build-up time jitter).

The pulse train widths of the hybrid mode-locked laser system $\left(T_{0}=0.7, V_{\mathrm{c}} \approx 50 \mathrm{~V}, P_{\mathrm{ao}} \approx 5 \mathrm{~W}\right)$ are $t_{1 / 2}(\mathrm{Nd}:$ phosphate $) \approx 150 \mathrm{~ns}$ and $t_{1 / 2}(\mathrm{Nd}$ : silicate $)$ $\approx 250 \mathrm{~ns}$.

\subsection{Output peak pulse intensity}

The output peak pulse intensities of the actively mode-locked double laser system at the first pulse train maxima are approximately $1.8 \times 10^{7} \mathrm{~W} / \mathrm{cm}^{2}$ for the Nd: phosphate laser branch and $2.2 \times 10^{7} \mathrm{~W} / \mathrm{cm}^{2}$ for the $\mathrm{Nd}$ : silicate laser branch at an excess flash- lamp pump voltage of $\mathrm{V}_{\mathrm{e}} \approx 300 \mathrm{~V}$ and acousto-optic mode-locker drive power of $P_{\mathrm{ao}} \approx 2 \mathrm{~W}$.

For the hybridly mode-locked laser system $\left(T_{0}=0.70 \mathrm{~V}_{\mathrm{e}}=50 \mathrm{~V}, P_{\mathrm{ao}}=5 \mathrm{~W}\right)$ the peak output pulse intensities at the pulse train maxima are $I_{\mathrm{OL}}$. $(\mathrm{Nd}$ : phosphate $) \approx 5 \times 10^{8} \mathrm{~W} / \mathrm{cm}^{2}$ and $I_{0 \mathrm{l}}$. $(\mathrm{Nd}$ : silicate $) \approx 1 \times 10^{9} \mathrm{~W} / \mathrm{cm}^{3}$.

\subsection{Spectral tuning}

The laser tuning of the active mode-locked double laser system is illustrated in fig. 4. The flashlamp voltages are kept $300 \mathrm{~V}$ above threshold. The azimuthal angle of the birefringent filters (thickness 5 $\mathrm{mm}$ ) is varied. The $\mathrm{Nd}$ : phosphate laser branch is tuned between $1048.5 \mathrm{~nm}$ and $1060 \mathrm{~nm}$ while the $\mathrm{Nd}$ : silicate laser branch is tuned between $1054.5 \mathrm{~nm}$ and $1072 \mathrm{~nm}$. The continuous frequency tuning range spans the region from $0-200 \mathrm{~cm}^{-1}$. The spectral widths of the pulses are $\Delta \tilde{\nu} \leqslant 1 \mathrm{~cm}^{-1}$.

For the hybrid mode-locked laser system the same tuning range is obtained $\left(T_{0} \approx 0.7, V_{\mathrm{e}} \approx 50 \mathrm{~V}, P_{\mathrm{ao}} \approx 5\right.$ W). The spectral width of the pulses broadens to $\Delta \tilde{\nu}_{1} \approx \Delta \tilde{\nu}_{2} \approx 20 \mathrm{~cm}^{-1}$. The spectral width of the pulses is expected to change along the pulse train. A more

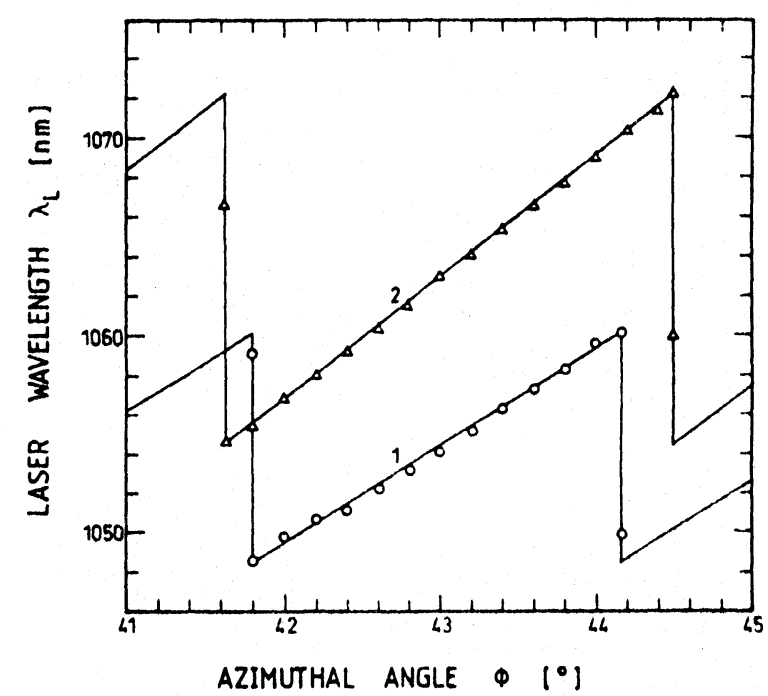

Fig. 4. Spectral tuning of acousto-optic mode-locked double laset system $\left(P_{\mathrm{ao}}=2 \mathrm{~W}\right)$. Curve 1 and circles: Nd:phosphate glass laser branch. Curve 2 and triangles: Nd silicate glass laser branch. The flashlamp excess pump voltage is $I_{c}^{\prime}=300 \mathrm{~V}$. 
definite situation would be obtained by single pulse selection.

\subsection{Temporal characteristics}

The pulse durations of the acousto-optic modelocked laser branches have been determined by intensity, energy, and beam cross-section measurement. At an excess flashlamp pump voltage of $V_{e} \approx 300 \mathrm{~V}$ (good time synchronization of both laser branches) and an acousto-optic mode-locker driver power of $P_{\mathrm{ao}} \approx 2 \mathrm{~W}$ pulse durations of the order of 200 ps were estimated.

The pulse durations of the hybrid mode-locked $\mathrm{Nd}$ : silicate laser branch have been measured applying the two-photon fluorescence technique. For $V_{\mathrm{e}} \approx 50 \mathrm{~V}, P_{\mathrm{ao}} \approx 5 \mathrm{~W}$, and $T_{0}($ No. 9740$)=0.7$, pulse durations of $\Delta t_{2}=15 \pm 3$ ps and contrast ratios of $2.7 \pm 0.3$ have been obtained. The threshold flashlamp voltages are $V_{\text {th }}(\mathrm{Nd}$ : phosphate $) \approx 1800 \mathrm{~V}$ and $V_{\text {th }}(\mathrm{Nd}$ : silicate $) \approx 1500 \mathrm{~V}$. Pumping the laser system with excess flashlamp voltages $V_{\mathrm{e}} \gtrsim 100 \mathrm{~V}$ resulted in multiple pulse emission within the envelope of the acousto-optic mode-locked pulse width. Small signal transmission $T_{0} \gtrsim 0.80$ of the saturable absorber have been found to be not sufficient to obtain good temporal overlap of the pulse trains of both branches for excess voltages $V_{e} \leqslant 50 \mathrm{~V}$.

The cross-correlation of the hybrid mode-locked laser system with $V_{\mathrm{e}}=50 \mathrm{~V}, P_{\mathrm{ao}}=5 \mathrm{~W}$, and $T_{0}$ (No. 9740 ) $=0.7$ was found to be $\Delta \tau_{\mathrm{c}} \approx 21 \pm 4 \mathrm{ps}$, indicating that the $\mathrm{Nd}$ :phosphate glass laser and the $\mathrm{Nd}$ : silicate glass laser give about the same pulse durations $\left(\Delta \tau_{\mathrm{c}}=\left[\left(\Delta t_{1}\right)^{2}+\left(\Delta t_{2}\right)^{2}\right]^{1 / 2}\right)$. For this experimental situation the synchronization jitter between the pulses of both trains was found to be $\sigma_{\mathrm{t}, \mathrm{p}} \approx 5 \mathrm{ps}$. The temporal pulse jitter is small compared to the temporal pulse width.

\section{Conclusions}

The performance of a time-synchronized picosecond double-frequency $\mathrm{Nd}$ :glass laser system has been investigated. Pulses of 15 ps duration with a synchronization jitter of 5 ps have been generated. The frequency difference between both lasers could be tuned continuously between 0 and $200 \mathrm{~cm}^{-1}$. The laser system may be applied to many experiments of time-resolved nonlinear optical spectroscopy where two intense laser pulses of slightly tunable wavelengths are needed.

\section{Acknowledgements}

The authors thank the Deutsche Forschungsgemeinschaft for financial support.

\section{References}

[1] G.R. Fleming, Chemical Applications of Ultrafast Spectroscopy (Oxford University Press, Oxford, 1986).

[2] A. Laubereau and M. Stockburger, eds. Time resolved vibrational spectroscopy, Proc. Phys. IV (Springer, Berlin. Heidelberg, 1985).

[3] W. Kaiser, ed., Ultrashort laser pulses and applications. Top. Appl. Phys., Vol. 60 (Springer, Berlin, Heidelberg, 1988 ).

[4] T. Yajima, K. Yoshihara, C.B. Harris and S. Shionoya, eds. Ultrafast Phenomena VI, Springer Ser. Chem. Phys., Vol. 48 (Springer, Berlin. Heidelberg, 1988).

[5] M.J. Wirth, Analytical Chemistr. 62 (1990) 270A.

16] Y.R. Shen, The principles of nonlinear optics (Wiley, New York, 1984).

[7] M. Schubert and B. Wilhelmi, Nonlinear optics and quantum electronics (Wiley, New York, 1986).

[8] S.A. Akhmanov, A.I. Kovrygin and A.P. Sukhorukov, in: Quantum electronics, Vol. 1, Part B, eds. H. Rabin and C.L. Tang (Academic Press. New York, 1975) p. 476.

[9] W. Kaiser and M. Maier, in: Laser Handbook, Vol. 2, eds. F.T. Arecchi and E.O. Schultz-Dubois (North-Holland, Amsterdam, 1972) Ch. E2.

[10] N. Bloembergen, Ann. J. Phys. 35 (1967) 989.

[11] A. Penzkofer, A. Laubereau and W. Kaiser, Prog. Quant. Electr. 6 (1979) 55.

[12] V. Wilke and W. Schmidt, Appl. Phys. 18 (1979) 177.

[13] L. Mannik and S.K. Brown, Optics Comm. 57 (1986) 360.

[14] A. Seilmeier, K. Spanner, A. Laubereau and W. Kaiser, Optics Conım. 24 ( 1978$) 234$.

[15] J.F. Reintjes, Nonlinear parametric processes in liquids and gases (Academic Press, Orlando, 1984).

[16] F. Wondrazek, A. Seilmeier and W. Kaiser, Appl. Phys. B32 (1983) 39

[17] T. Elsaesser, H.J. Polland, A. Seilmeier and W'. Kaiser, IEEE J. Quant. Electr. QE-20 (1984) 191.

[18] S. Szatmari and F.P. Schäfer, Optics Comm. 49 (1984) 281.

[19] Zs. Bor, S. Szatmari and A. Müller, Appl. Phys. B32 (1983) 101.

[20] P. Sperber, W. Sprangler, B. Meier and A. Penzkofer, Opt. Quant. Electr. 20 (1988) 395.

[21] P. Qiu and A. Penzkofer, Opt. Quant. Electr. B48 (1989) 115. 
[22] A. Penzkofer and A.A. Bugayev, Opt. Quant. Electr. 21 (1989) 283

[23] J. Hebling and J. Kuhl, Optics Comm. 73 (1989) 375.

[24] L.S. Goldberg and C.A. Moore, Appl. Phys. Lett. 27 (1975) 217.

[25] J.P. Heritage and R. Jain, Appl. Phys. Lett. 32 (1978) 101.

[26] J. Kuhl, Appl. Phys. B28 (1982) 259.

[27] H. Lobentanzer, Optics Comm. 71 (1989) 175.

[28] U. Siegner. G. Noll and E.O. Göbel, Appl. Phys. B48 (1989) 21.

[29] A. Laubereau, in: Ultrashort lasers and applications, ed. W. Kaiser, Top. Appl. Phys., Vol. 60 (Springer, Berlin, Heidelberg. 1988) p. 35.

[30] R.R. Alfano, ed., The super continuum laser source (Springer, Berlin, Heidelberg, 1989).

[3] ] D.R. Preuss and J.L. Gole, Appl. Optics 19 (1980) 702

[32] A.E. Siegman, IEEE J. Quant. Electr. QE-9 (1973) 247.

[33] R. Trutna and A.E. Siegman, IEEE J. Quant. Electr. QE-13 (1977) 955 .

[34] H. Vanherzeele, J.L. Van Eck and A.E. Siegman, Appl. Optics 20 (1981) 3484.

[35] W. Koechner. Solid-state laser engineering, Springer Series in Opt. Sciences, Vol. 1, 2nd revised Edition (Springer, Berlin. Heidelberg, 1988).

[36] G.F. Albrecht, L. Lund and D. Smith, Appl. Optics 22 (1983) 1276.

[37] A. Penzkofer. Appl. Phys. B46 (1988) 43.

[38] G. Bergamasco and R. Polloni, Optics Lett. 11 (1986) 222.

[39]A.E. Siegman and H. Vanherzeele, in: Picosecond phenomena III, Springer Ser. in Chem. Phys., Vol. 23, eds. K.B. Eisenthal, R.M. Hochstrasser, W. Kaiser and A. Laubereau (Springer, Berlin, Heidelberg, 1982) p. 14.

[40] J.M. Buchert, A.K. Basa, C. Tzu and R.R. Alfano, J. Appl. Phys. 55 (1984) 683.

[41] L. Lin, G. Sheng, W. Zhang and Y. Kang. Chin. Phys. Lett. 3 (1986) 329.

[42] V.I. Prokhonenko, E.A. Tikhonov. D.Ya. Yatskiv and E.N. Bushmakin. Sov. J. Quant. Electron. 17 (1987) 505.

[43] A. Daugvila, A. Deringas, V. Kabelka, A.V. Masalov and A. Miliauskas, in: Ultrafast phenomena in spectroscopy, eds. Z. Rudzikas, A. Piskarskas and R. Baltramiejūnas (World Scientific, Singapore, 1988) p. 56.

[44] A. Daugvila, V. Kabelka and A.V. Masalov, Sov. J. Quant. Electr. 18 (1988) 920.
[45] X. Lin, W.Z. Zhang, J.X. Wang, F.T. Wu and Z.A. Sun. Optics Comm. 71 (1989) 367.

[46] G.P. Banfi, G. Gabetta, P.G. Gobbi and G.C. Reali, Proc. VI. Intern. Symposium Ultrafast Phenomena in Spectroscopy, 1989, Neubrandenburg, GDR (SpringerVerlag, Berlin, Heidelberg) to be published.

[47] S.V. Vinogradev, V.V. Kuznetsov, B.S. Neporent and V.B. Shilov, in: Proc. VI. Intern. Symposium Ultrafast Phenomena in Spectroscopy, 1989, Neubrandenburg, GDR (Springer-Verlag, Berlin, Heidelber'́) to be published.

[48] A. Daugvila, V. Kabelka and A.V. Masalov, in: Proc. of VI Int. Symposium Ultrafast Phenomena in Spectroscopy, 1989. Neubrandenburg, GDR (Springer-Verlag. Berlin. Heidelberg) to be published.

[49] M.I. Demchuk, V.P. Kalosha, V.P. Mikhailov and I.A. Manichev, Opt. Quant. Electr. 21 (1989) 481.

[50] J.C. Diels, I.C. McMichael, F. Simoni, R. Torti, H. Vanherzeele, W. Dietel, E. Döpel, W. Rudolph, B. Wihelmi and J. Fontaine, in: Ultrafast Phenomena IV, Springer Ser. in Chemical Phys., Vol. 38, eds. D.H. Auston and K.B. Eisenthal (Springer, Berlin, Heidelberg, 1984) p. 30.

[51] H. Vanherzeele, J.-C. Diels and R. Torti, Optics Lett. 9 (1984) 549.

[52] J.C. Diels, N. Jamashi and L. Sarger, in: Ultrafast Phenomena V, Springer Ser. in Chem. Phys., Vol. 46, eds. G.R. Flemming and A.E. Siegman (Springer, Berlin, Heidelberg. 1986) p. 2.

[53] H. Schillinger and A. Penzkofer, Optics Comm. 68 (1988) 45.

154] A. Penzkofer, A. Laubereau and D. von der Linde, Optics Comm. 4 (1972) 377

[55] J.A. Giordmaine, P.M. Rentzepis, S.L. Shapiro and K.W. Wecht, Appl. Phys. Lett. 11 (1967) 216.

[56] E.P. Ippen and C.V. Shank, in: Ultrafast Light Pulses, ed. S.L. Shapiro, Top. in Appl. Phys., Vol. 18 (Springer, Berlin, Heidelberg, 1977) p. 83.

[57] P. Sperber and A. Penzkofer, Opt. Quant. Electr. 18 (1986) 145.

[58] A. Penzkofer and F. Graf, Opt. Quant. Electr. 17 (1985) 219.

[59] A. Penzkofer, Prog. Quant. Electron. 12 (1988) 291.

[60] F. Graf and A. Penzkofer, Opt. Quant. Electr. 17 (1985) 53.

[61] D. von der Linde and K.F. Rodgers, IEEE J. Quant. Electr. QE-9 (1973) 960 